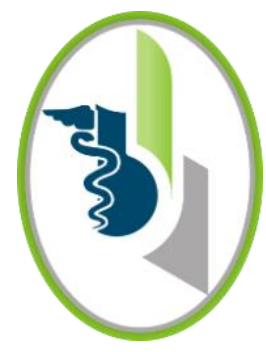

ACCESO abierto

Para citaciones: Cano, L., Muñoz, L., Durán, M. (2019). Resultados clínicos luego de la administración de albúmina en pacientes con desnutrición severa. Revista Ciencias Biomédicas, 8(2), 51-59.

Recibido: 29 de enero de 2019

Aprobado: 23 de mayo de 2019

Autor de correspondencia:

Larissa Cano Duran

mduranl@unicartagena.edu.co

Editor: Inés Benedetti. Universidad de Cartagena-Colombia.

Copyright: (C) 2019. Cano, L., Muñoz, L. Durán, M. Este es un artículo de acceso abierto, distribuido bajo los términos de la licencia https://creativecommons.org/licenses/by-nc-

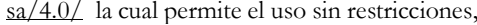
distribución y reproducción en cualquier medio, siempre y cuando el original, el autor y la fuente sean acreditados.

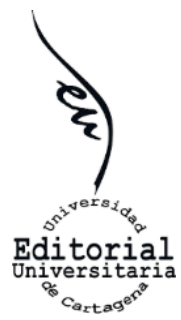

\section{Resultados clínicos luego de la administración de albúmina en pacientes con desnutrición severa}

\author{
Clinical results after albumin administration in patients with \\ severe malnutrition.
}

Cano Durán Larissa ${ }^{1}$, Muñoz Álvarez Nelson², Durán Lengua Marlene ${ }^{3}$

${ }^{1}$ Hospital Infantil Napoléon Franco Pareja, Cartagena, Colombia

${ }^{2}$ Departamento de Pediatría, Facultad de Medicina, Universidad de Cartagena, Colombia

${ }^{3}$ Maestría en Farmacología, Facultad de Medicina, Universidad de Cartagena

\section{RESUMEN}

Introducción: la desnutrición infantil sigue siendo un problema de gran magnitud a nivel mundial, fundamentalmente para la población menor de 5 años en los países en vías de desarrollo, constituyendo un tercio del total de las causas de muertes en niños.

Objetivo: describir los resultados clínicos de la aplicación de albúmina en pacientes con desnutrición severa en el Hospital Infantil Napoleón Franco Pareja (HINFP).

Métodos: se realizó un estudio prospectivo en el cual participaron pacientes pediátricos que asistieron al HINFP, se valoró su estado nutricional mediante medidas antropométricas y marcadores clínicos como frecuencia cardíaca y respiratoria, tensión arterial, estancia hospitalaria, ingreso a UCI y muerte. Las evaluaciones fueron seriadas durante la estancia hospitalaria para describir la evolución clínica secundaria a manejo intrahospitalario con albúmina. Las comparaciones entre los grupos se hicieron a través de la prueba Chi2 o el test de Fisher, con el programa Epi Info V.7.2.0.1.

Resultados: se analizaron 25 pacientes pediátricos, 56\% de sexo masculino. El tratamiento con albúmina fue administrado al $48 \%$ de los pacientes, dentro de los signos de desnutrición severa el edema se observó en el 91,7\% de los pacientes tratados con albúmina y en el grupo no tratado fue del $46.2 \%, \mathrm{p}=0,0446$, la emaciación fue observada entre los grupos en $16,7 \%$ y $30,8 \%$ respectivamente, $\mathrm{p}=, 07219$, el $100 \%$ de los pacientes egresaron vivos.

Conclusión: no se evidenciaron diferencias signifcativas entre los pacientes tratados con albúmina y quienes no se le administró el tratamiento, se recomienda realizar estudios con una muestra mayor.

Palabras Clave: Desnutrición infantil; albúmina; desnutrición proteica.

\section{ABSTRACT}

Introduction: child malnutrition continues to be a problem of great magnitude worldwide, mainly for the population under 5 years of age in developing countries, constituting a third of the total causes of death in children. 
Objective: to estimate the effectiveness of the application of albumin in patients with severe malnutrition at the Napoleón Franco Pareja Children's Hospital (HINFP).

Methods: a prospective study was conducted in which pediatric patients attended the HINFP participated. The nutritional status of the patient was assessed by means of anthropometric measures, and clinical markers such as heart rate and respiration, blood pressure, hospital stay, ICU admission and death. These tests were performed in a serial manner during the hospital stay for the description of evolution secondary to intrahospitalary management with albumin. For the comparison of results, the $\mathrm{Chi}^{2}$ and Fisher test were used. The statistical analysis was carried out using the EpiInfo V.7.2.0.1.

Results: twenty-five pediatric patients participated, 56 male. Treatment with albumin was administered to $48 \%$ of the patients, within the signs of severe malnutrition the edema was observed in $91.7 \%$ of the patients treated with albumin and in the untreated group was $46.2 \%, p=0,0446$, the emaciation was observed between the groups in $16.7 \%$ and $30.8 \%$ respectively, $\mathrm{p}=, 07219.100 \%$ of the patients were alive.

Conclusions: there were no differences in the patients treated with albumin and those who were not treated, it is recommended to perform studies with a larger sample.

Key Words: Child malnutrition; albumin; protein malnutrition.

\section{INTRODUCCIÓN}

La desnutrición infantil sigue siendo un problema de gran magnitud a nivel mundial, fundamentalmente para la población menor de 5 años en los países en vías de desarrollo, constituyendo un tercio del total de las causas de muertes en niños (1). En el 2013, 51 millones de niños presentaron desnutrición moderada y 17 millones desnutrición severa (1). La desnutrición severa ha causado 1.5 millones de muertes anuales en los últimos 5 años, por lo que la mejoría de la supervivencia es uno de los principales objetivos en salud pública, constituido como el cuarto entre los objetivos de los desarrollos del milenio para disminuir la mortalidad infantil (2).

En Colombia existen regiones con tasas de desnutrición infantil semejantes a algunas zonas de África, como el departamento del Vaupés, donde la desnutrición infantil crónica afecta al 34,7\% de los menores de 5 años y el departamento de La Guajira, donde tal drama lo vive el 27,9\% de la población infantil (3). Por su parte el departamento de Bolívar reporta porcentajes del $11.5 \%$. Sumado a lo anterior, desde hace ya varios años la Sociedad Colombiana de Pediatría ha denunciado que la verdadera causa de muchas de las muertes en niños en el territorio nacional es debido a la desnutrición, pero dicho motivo queda enmascarado en diagnósticos tales como neumonías, diarreas, infecciones graves, entre otros, que a su vez pueden devenir por causa de la misma (4). Es decir, en el territorio colombiano sigue existiendo un subregistro en las muertes de niños por problemas asociados a las carencias nutricionales, lo que demuestra el desconocimiento de la magnitud de esta problemática en salud pública (4).

Una de las principales consecuencias de la desnutrición infantil es la disminución cuantitativa de los niveles de proteínas en plasma sanguíneo como la albúmina, lo que genera un estado de disfunción orgánica que varía inversamente al aporte nutricional que se pueda suplir con los recursos disponibles (5). La albúmina es una proteína encargada del mantenimiento de la presión oncótica en el espacio vascular y del transporte de 
sustancias plasmáticas; cuando esta se encuentra disminuida, se produce una alteración en la distribución de los líquidos corporales, causando extravasación y edema, lo que se asocia a hipovolemia e inestabilidad hemodinámica (6). Por esta explicación fisiopatológica se ha intentado demostrar la eficacia clínica de la administración de albúmina como reemplazo de las carencias nutricionales, para proveer una función plasmática adecuada en medio del estado hipercatabólico del niño con desnutrición (7). A pesar de que los resultados de los estudios con aplicación de albúmina en población adulta con desnutrición han sido controversiales y desalentadores, en la población pediátrica los estudios al respecto son pocos con pequeños tamaños de muestra lo que limita la validez externa de los mismos (8).

No obstante, a la controversia del uso de la albúmina en niños, esta sigue siendo parte del tratamiento de la desnutrición global severa en muchas instituciones de salud que tratan a población infantil, como es el caso del Hospital Infantil Napoleón Franco Pareja (HINFP) de la ciudad de Cartagena, Colombia. Al no poder generalizar los resultados de la literatura mundial del uso de la albúmina en nuestros pacientes, se planteó la necesidad de realizar el presente estudio que tuvo como objetivo describir los resultados clínicos luego de la administración de albúmina en pacientes pediátricos con desnutrición severa.

\section{MÉTODOS}

Se realizó un estudio observacional descriptivo, de carácter prospectivo, en donde se tomó como población de estudio a todos los pacientes pediátricos entre los 0 meses y 5 años de edad que asistieron al HINFP que cumplieran algún criterio de desnutrición global severa como: emaciación visible, peso/talla por debajo de tres desviaciones estándar, peso/edad por debajo de tres desviaciones estándar, y edema pedio bilateral. Fueron excluidos los pacientes con desnutrición global severa que tuvieran además alguna enfermedad de base como neuropatías, miopatías, cardiopatías, malformaciones somáticas severas, enfermedades gastrointestinales y genéticas que sean predisponentes de su estado de desnutrición.

El primer día de hospitalización se registraron variables como edad, sexo y administración de albúmina como tratamiento, además se indagaron antecedentes perinatales como: semanas de edad gestacional, peso en gramos al nacimiento, realización de buen control prenatal (definido como el cumplimiento de cuatro citas o más), clasificación neonatal del recién nacido teniendo en cuenta su condición de pretérmino (RNPr), a término (RNT) y postérmino (RNPt), y el peso en: pequeño (PEG), adecuado (AEG) y grande para edad gestacional (GEG), además, la necesidad de reanimación neonatal.

Dentro de las variables clínicas de ingreso se tuvo en cuenta el peso, talla e índice de masa corporal, la frecuencia cardiaca, frecuencia respiratoria, llenado capilar, presencia de edema pedio bilateral, emaciación visible y signos secundarios de desnutrición como dermatosis, signo de la bandera, cabello quebradizo, atrofia muscular, entre otros. La valoración de la evolución clínica tomó como variables de seguimiento la frecuencia cardiaca, frecuencia respiratoria, llenado capilar, presencia de edema pedio bilateral identificada el último día de hospitalización previo al egreso, además se describió la necesidad de manejo en unidad de cuidados intensivos pediátrica (UCIP), estado al egreso tomado como vivo o muerto, los días de estancia hospitalaria totales (Incluyendo la de UCIP), y la conducta extrahospitalaria tomada.

\section{Análisis estadístico}

El análisis estadístico de las variables cualitativas se realizó mediante el cálculo de frecuencias absolutas y relativas; por su parte las variables cuantitativas se analizaron con mediana (Me) y rango intercuartílico (RIC), por la naturaleza no paramétrica de todas las variables estimada por prueba de Shapiro Wilk. Para realizar las comparaciones entre los grupos de pacientes que recibieron o no tratamiento con albúmina se utilizó la prueba $\mathrm{Chi}^{2}$ o el test de Fisher según fuera necesario en las variables cualitativas, y el test de Mann Whitney en las cuantitativas. Un valor de $\mathrm{p}<0,05$ fue considerado estadísticamente 
significativo, este análisis se realizó con el programa Epi Info ${ }^{\circledR}$ V.7.2.0.1.

\section{RESULTADOS}

En total se identificaron 211 pacientes con diagnóstico de algún grado de desnutrición en el periodo de estudio. De estos se excluyeron 94 por presentar comorbilidades predisponentes de su estado nutricional y 92 por presentar desnutrición leve o moderada, en total se analizaron 25 pacientes pertenecientes al estrato socioeconómico 1. De estos la distribución por sexo mostró una mayor frecuencia de niños con $56 \%$, la mediana de edad general fue de 12 meses (RIC 8 - 12), el tratamiento con albúmina fue administrado al $48 \%$ de la muestra correspondiente a 12 pacientes, (Tabla 1).

Tabla 1. Características sociodemográficas de los pacientes

\begin{tabular}{ccc}
\hline & $\mathrm{n}$ & $\%$ \\
\hline Sexo & & 44.0 \\
\hline Femenino & 11 & 56,0 \\
\hline Masculino & 14 & \\
\hline Edad Me (RIC) & $12(8-12)$ & 40,0 \\
\hline Lactante menor & 10 & 44,0 \\
\hline Lactante mayor & 11 & 16,0 \\
\hline Preescolar & 4 & 48,0 \\
\hline
\end{tabular}

Al comparar los antecedentes perinatales de los pacientes estudio por administración de albúmina o no, se observó una mediana de edad gestacional al nacimiento de 39 semanas en ambos grupos, con mediana de peso de 3400 en los que recibieron albúmina y 3500 en aquellos sin el medicamento. La realización de un buen control prenatal se encontró en $25 \%$ y $7,7 \%$ respectivamente entre los pacientes con y sin administración de albúmina; por su parte la clasificación neonatal de los pacientes al nacimiento fue similar en los grupos de estudio excepto en los recién nacidos a término adecuados para la edad gestacional (RNTAEG) que se encontraron en $41,7 \%$ en el grupo de albúmina comparado con ningún paciente en el grupo no tratado, $(p=0,035)$. El antecedente de necesidad de reanimación fue descrito en el $8,3 \%$ de los pacientes con albúmina y en ninguno sin el medicamento, (Tabla 2).

El cuadro clínico de ingreso mostró en cuanto el peso, talla e IMC medianas similares entre los grupos con y sin administración de albúmina. Por su parte los signos vitales como la frecuencia cardiaca presentó una mediana de 139 lpm $(\mathrm{RIC}=120$ - 147) y $120 \quad(\mathrm{RIC}=106$ - 123) lpm en los grupos respectivamente, $\quad(p=0,048) ; \quad$ la frecuencia respiratoria tuvo medianas respectivas en los grupos de 37 y $36 \mathrm{rpm},(\mathrm{p}=0,956)$. El llenado capilar fue de menos de 2 segundos en al menos el $75 \%$ en ambos grupos, seguido de menor de 3 segundos en $16,7 \%$ de los pacientes con albúmina y $7,7 \%$ en los pacientes sin ella, en al menos el 7,7\% de ambos grupos el llenado capilar fue mayor a 3 segundos. 
Tabla 2. Antecedentes perinatales de los pacientes

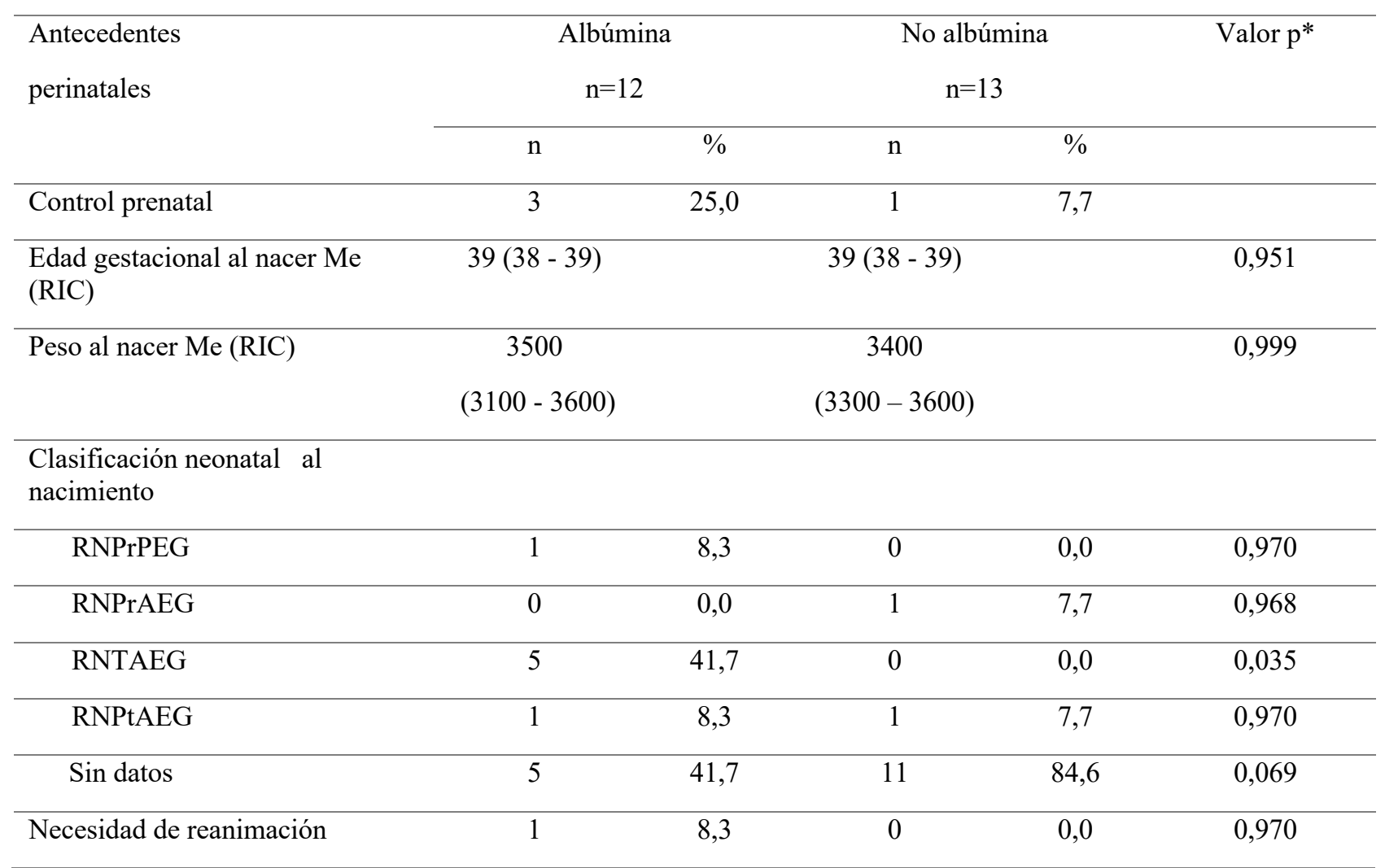

* Prueba $\mathrm{Chi}^{2}$

Dentro de los signos de desnutrición severa el edema se observó en el $91,7 \%$ de los pacientes tratados con albúmina y en el grupo no tratado fue del $46.2 \%$, $(p=0,044)$; la emaciación fue observada entre los grupos en $16,7 \%$ y $30,8 \%$ respectivamente, $(p=, 0721)$. Los signos secundarios de desnutrición más frecuentemente observados en los grupos con y sin albúmina fueron en orden de frecuencia: la dermatosis diseminada en el $69,2 \%$ del grupo sin albúmina $\mathrm{y}$ en $100 \%$ de los tratados con el medicamento, signo de la bandera en más del $50 \%$ en ambos grupos, dermatitis del pañal y queilitis angular en más del $37 \%$ en ambos grupos, pelagra y cabello quebradizo en más del $46 \%$, (Tabla 3 ).

La evolución clínica de los pacientes según el tratamiento suministrado mostró en los grupos con y sin albúmina una mediana de frecuencia cardiaca de 114 y 112 lpm, de frecuencia respiratoria de 30 y 33 rpm respectivamente, y en más del $76 \%$ de los casos en ambos grupos un llenado capilar menor de 2 segundos. Por otro lado, el edema al egreso se observó en $66,7 \%$ de los pacientes con albúmina y en $23,1 \%$ en los pacientes no tratados con ella, $(\mathrm{p}=0,183)$, observándose una mejoría del edema en los grupos del $25 \%$ y $23,1 \%$ respectivamente, $(\mathrm{p}=0,959)$. La remisión a UCIP fue necesaria en $8,3 \%$ de los pacientes con administración de albúmina y en $23,1 \%$ de los pacientes en los que no se usó, $(p=0,330)$. El $100 \%$ de los pacientes egresaron vivos del HINFP encontrándose una mediana de días de estancia hospitalaria de 10 días $(\mathrm{RIC}=8-17)$ en los que fueron tratados con albúmina, y de 6 días $(\mathrm{RIC}=3-10)$ en los que no se usó, $(p=0,008)$. Las conductas extrahospitalarias al egreso fueron en primer lugar el seguimiento por consulta externa en el $83,3 \%$ y $76,9 \%$ de los grupos con y sin albúmina, $y$ en $16,7 \%$ y $23,1 \%$ respectivamente hubo derivación a un centro de recuperación nutricional, $(\mathrm{p}=0,919)$, (Tabla 4$)$. 
Tabla 3. Cuadro clínico al ingreso de los pacientes

\begin{tabular}{|c|c|c|c|c|c|}
\hline \multirow[t]{2}{*}{ Cuadro clínico (ingreso) } & \multicolumn{2}{|c|}{$\begin{array}{l}\text { Albúmina } \\
(\mathrm{n}=12)\end{array}$} & \multicolumn{2}{|c|}{$\begin{array}{l}\text { No albúmina } \\
\qquad(\mathrm{n}=13)\end{array}$} & \multirow[t]{2}{*}{ Valor $\mathrm{p}^{*}$} \\
\hline & $\mathrm{n}$ & $\%$ & $\mathrm{n}$ & $\%$ & \\
\hline Peso en kg ME (RIC) & 6,8 & $(5,9-7.9)$ & 6,3 & $(6,0-7.8)$ & 0,585 \\
\hline Talla en cm ME (RIC) & 68,5 & $(63,5-74,0)$ & 70,0 & $(64,0-75,0)$ & 0,913 \\
\hline IMC ME (RIC) & 13,8 & $(13,2-16,3)$ & 12,6 & $(11,8-13,9)$ & 0,072 \\
\hline $\begin{array}{l}\text { Frecuencia cardiaca } \\
\text { ME (RIC) }\end{array}$ & 139 & $(120-147)$ & 120 & $(106-123)$ & 0,048 \\
\hline $\begin{array}{l}\text { Frecuencia respiratoria } \\
\qquad \mathrm{ME}(\mathrm{RIC})]\end{array}$ & 37 & $(29-48)$ & 36 & $(32-42)$ & 0,956 \\
\hline \multicolumn{6}{|l|}{ Llenado capilar } \\
\hline Menor a 2 segundos & 9 & 75,0 & 11 & 84,6 & 0,921 \\
\hline Menor a 3 segundos & 2 & 16,7 & 1 & 7,7 & 0,939 \\
\hline Mayor a 3 segundos & 1 & 8,3 & 1 & 7,7 & 0,494 \\
\hline Edema & 11 & 91,7 & 6 & 46,2 & 0,044 \\
\hline Emaciación visible & 2 & 16,7 & 4 & 30,8 & 0,721 \\
\hline \multicolumn{6}{|l|}{ Signos secundarios } \\
\hline Cabello quebradizo & 8 & 66,7 & 6 & 46,2 & 0,529 \\
\hline Signo de la bandera & 10 & 83,3 & 7 & 53,9 & 0,252 \\
\hline Dermatosis diseminada & 12 & 100,0 & 9 & 69,2 & 0,120 \\
\hline Dermatitis del pañal & 10 & 83,3 & 5 & 38,5 & 0,060 \\
\hline Queilitis angular & 10 & 83,3 & 5 & 38,5 & 0,060 \\
\hline Pelagra & 9 & 75,0 & 6 & 46,2 & 0,289 \\
\hline Manifestaciones neurológicas & 0 & 0,0 & 1 & 7,7 & 0,968 \\
\hline Atrofia muscular & 0 & 0,0 & 1 & 7,7 & 0,968 \\
\hline Rosario costal & 0 & 0,0 & 1 & 7,7 & 0,968 \\
\hline Hepatoesplenomegalia & 0 & 0,0 & 1 & 7,7 & 0,968 \\
\hline
\end{tabular}

* Prueba $\mathrm{Chi}^{2}$ 
Tabla 4. Tratamiento suministrado y evolución clínica de los pacientes

\begin{tabular}{|c|c|c|c|c|c|}
\hline & \multicolumn{2}{|c|}{$\begin{array}{l}\text { Albúmina } \\
\qquad(\mathrm{n}=12)\end{array}$} & \multicolumn{2}{|c|}{ No albúmina $(\mathrm{n}=13)$} & \multirow[t]{2}{*}{ Valor $\mathrm{p}^{*}$} \\
\hline & $\mathrm{n}$ & $\%$ & $\mathrm{n}$ & $\%$ & \\
\hline \multicolumn{6}{|l|}{ Valoración postratamiento } \\
\hline Frecuencia cardiaca & 114 & $(111-125)$ & 112 & $(98-124)$ & 0,312 \\
\hline \multicolumn{6}{|l|}{ ME (RIC) } \\
\hline Frecuencia respiratoria & 30 & $(28-34)$ & 33 & $(32-40)$ & 0,070 \\
\hline \multicolumn{6}{|l|}{$\mathrm{ME}(\mathrm{RIC})]$} \\
\hline \multicolumn{6}{|l|}{ Llenado capilar } \\
\hline Menor a 2 segundos & 11 & 91,7 & 10 & 76,9 & 0,643 \\
\hline Menor a 3 segundos & 0 & 0,0 & 0 & 0,0 & -- \\
\hline Mayor a 3 segundos & 1 & 8,3 & 3 & 23,1 & 0,330 \\
\hline Edema & 8 & 66,7 & 3 & 23,1 & 0,183 \\
\hline Desaparece edema & 3 & 25,0 & 3 & 23,1 & 0,959 \\
\hline Estancia hospitalaria (días) & 10 & $(8-17)$ & 6 & $(3-10)$ & 0,008 \\
\hline \multicolumn{6}{|l|}{$\mathrm{Me}(\mathrm{RIC})$} \\
\hline Remisión a UCIP & 1 & 8,3 & 3 & 23,1 & 0,330 \\
\hline Inotrópicos & 1 & 8,3 & 2 & 15,4 & 0,944 \\
\hline Estado al egreso vivo & 12 & 100,0 & 13 & 100,0 & -- \\
\hline \multicolumn{6}{|l|}{ Conducta extrahospitalaria } \\
\hline Seguimiento por consulta externa & 10 & 83,3 & 10 & 76,9 & 0,919 \\
\hline Centro de recuperación nutricional & 2 & 16,7 & 3 & 23,1 & \\
\hline
\end{tabular}

* ${\text { Prueba } \mathrm{Chi}^{2}}^{2}$

\section{DISCUSIÓN}

La desnutrición infantil es uno de los problemas más comunes en la infancia, presente en los países en vía de desarrollo, siendo la desnutrición proteicocalórica una de las relevantes, la cual produce disminución de la disponibilidad de proteínas en 
especial la albúmina (9). En el presente estudio se incluyeron pacientes pediátricos en su mayoría de sexo masculino y que además vivían en un estrato socioeconómico bajo, datos similares a los reportados por Demisse y Sobrino donde se evidenció que los pacientes que presentaban desnutrición crónica eran principalmente del sexo masculino $(10,11)$. Esta mayor frecuencia en la población masculina puede ser debida a que existen desigualdades en salud entre niños y niñas recibiendo estas últimas una mayor atención (10). Sobrino además encontró que estos pacientes provenían de familias con ingresos bajos, lo que refuerza la premisa que la pobreza es considerada como un determinante en la desnutrición e inseguridad alimentaria, siendo este un sígno de desigualdad económica y social $(10,12)$.

El bajo peso al nacer es considerado por la OMS como el peso al nacimiento menor a 2500 gramos, este se convierte en un determinante de riesgo para el desarrollo de malnutrición infantil, al estar estos niños en desventajas generando aumento en la probabilidad de enfermar y morir, debido que requieren de más cuidados para poder mantener un adecuado estado de salud (13) (14). Silveira encontró que los pacientes que presentaban desnutrición crónica se encontraban asociados a un peso al nacer menor de 2500 gramos, un nivel socioeconómico bajo y una introducción inadecuada de la alimentación complementaria (15). En el presente estudio no se evidenció una alta frecuencia del antecedente de bajo peso al nacer.

En cuanto a las manifestaciones clínicas que presentaron nuestros infantes al ingreso fueron principalmente edema, dermatosis y signo de la bandera, estos datos concuerdan con los evidenciados en el estudio realizado por Bilbao y cols, en el cual las características clínicas que presentaban los pacientes eran alteraciones cutáneas y de mucosas, edema y alteraciones evidentes en la masa muscular (16). En Bolivia, Weisstaub y cols, encontraron que la mayoría de los niños hospitalizados por signos de desnutrición presentaron edema y diarrea, además de lesiones cutáneas (17). La presencia de edema ocurre principalmente por el déficit de proteínas como la albúmina, lo que conlleva a una disminución de la presión oncótica, además de la incapacidad de activación de la hormona antidiurética, y produce aumento de líquido en el espacio intersticial (18).

El tratamiento de los niños con desnutrición se basa en la complementación de las necesidades energéticas del paciente con una dieta apropiada para su edad, manejo de la alimentación y en algunos casos suplemento de vitaminas y minerales (19). En algunos casos se recomienda la reposición de proteínas como la albúmina, en este estudio no se observó una diferencia en la evolución clínica de los pacientes en los signos clínicos como el llenado capilar, las frecuencias cardiaca y respiratoria, pero principalmente en el edema pedio bilateral, con un aumento significativo de la estancia hospitalaria en el grupo tratado con albúmina. Estos hallazgos son consistentes con lo descrito por Porto que evaluó el uso de albúmina en recién nacidos críticamente enfermos sin encontrar su utilidad como método de nutrición parenteral (20); pero difiere de lo descrito en Colombia por León quien si describió una mejoría de los síntomas al ingreso de los pacientes desnutridos (19). El hallazgo relacionado con el aumento de la estancia hospitalaria en el grupo manejado con albúmina puede deberse a que el manejo con este medicamento no está protocolizado en la institución y su uso se restringe a criterio del pediatra tratante (7), pudiendo esto sesgar su utilización a aquellos pacientes que clínicamente se encuentran en peor estado y que requieren de mayores cuidados hospitalarios, hecho que se refleja en los días de estancia hospitalaria. A pesar de la controversia entre el uso o no de albúmina en el manejo de la desnutrición en el paciente pediátrico, esta constituye una opción terapéutica.

El presente estudio sugiere que la evolución clínica de los pacientes pediátricos con desnutrición severa tratados con albúmina no difiere de aquellos en los que no se usa el medicamento. Sin embargo, al tener este estudio la limitación de un número reducido de pacientes, resultado de los estrictos criterios de selección utilizados, se requieren estudios cuya duración sea más prolongada, que permitan la 
captación y seguimiento de un mayor número de pacientes, para así evaluar de manera precisa su efectividad.

\section{CONFLICTO DE INTERESES: Ninguno que declarar}

\section{REFERENCIAS}

1. Kramer CV, Allen S. Malnutrition in developing countries. Paediatrics and Child Health. 2015;25(9):422-7.

2. Fergusson P, Tomkins A, Kerac M. Improving survival of children with severe acute malnutrition in HIVprevalent settings. International Health. 2009;1(1):10-6.

3. Fonseca Z, Heredia A, Ocampo P, Forero Y, Sarmiento O, Álvarez M, et al. Encuesta nacional de la situación nutricional en Colombia 2010. Bogotá: Ministerio de la Protección Social. 2011.

4. Sociead Colombiana de Pediatría, Comité de Pediatría Social y Derechos. Mortalidad y desnutrición en los niños y niñas en Colombia: una realidad inaceptable. Comunicado Semana epidemiológica 04. 2016.

5. Levitt DG, Levitt MD. Human serum albumin homeostasis: a new look at the roles of synthesis, catabolism, renal and gastrointestinal excretion, and the clinical value of serum albumin measurements. International journal of general medicine. 2016;9:229.

6. Quinlan GJ, Martin GS, Evans TW. Albumin: biochemical properties and therapeutic potential. Hepatology. 2005;41(6):1211-9.

7. Uhing MR. The albumin controversy. Clinics in perinatology. 2004;31(3):475-88.

8. Macallan D. Infection and malnutrition. Medicine. 2009;37(10):525-8.

9. Ramos-Martínez K, González-Martínez F, LunaRicardo L. Estado de salud oral y nutricional en niños de una institución educativa de Cartagena, 2009. Revista de Salud Pública. 2010;12:950-60.

10. Sobrino M, Gutiérrez C, Cunha AJ, Dávila M, Alarcón J. Desnutrición infantil en menores de cinco años en Perú: tendencias y factores determinantes. Rev. Panam. Salud Pública. 2014;35(2):104-12.

11. Demissie S, Worku A. Magnitude and factors associated with malnutrition in children 6-59 months of age in pastoral community of Dollo Ado district, Somali region, Ethiopia. Sci J Public Health. 2013;1(4):175-83.

12. Rajmil L, Díez E, Peiró R. Desigualdades sociales en la salud infantil. Informe SESPAS 2010. Gaceta Sanitaria. 2010;24:42-8.

13. Kac G, García Alvear J. Epidemiología de la desnutrición en Latinoamérica: situación actual. Nutrición Hospitalaria. 2010;25.

14. Rodríguez SO, Medina MM, González AR. Determinantes de la desnutrición infantil en el municipio Camagüey. Revista Cubana de Alimentación y Nutrición. 2014;24(1):12.

15. Silveira K, Alves J, Ferreira H, Sawaya A, Florêncio T. Association between malnutrition in children living in favelas, maternal nutritional status, and environmental factors. Jornal de pediatria. 2010;86(3):21520.

16. Bilbao-González K, Martell-Betancourt NL, Gómez-López L, García-Padrón J, Segredo-Molina Y, González-Luzardo W. Características clínicas y evolución de niños con desnutrición aguda. Acta Médica del Centro. 2012;6(3):17-23.

17. Weisstaub G, Soria R, Araya M. Desnutrición grave en un hospital pediátrico. Revista de la Sociedad Boliviana de Pediatría. 2006;45(2):90-4.

18. Cohendy R, Rubenstein L, Eledjam J. The Mini Nutritional Assessment-Short Form for preoperative nutritional evaluation of elderly patients. Aging Clinical and Experimental Research. 2001;13(4):293-7.

19. León Acevedo L, Latorre Latorre J, Rodríguez Moncada M. Uso de albúmina humana en desnutrición edematosa. Revista de la Universidad Industrial de Santander Salud. 2007;39(2).

20. Porto B, Jorge S, de Assis M. Exogenous human albumin supplementation in total parenteral nutrition of critically ill newborns. J Pediatr (Rio J). 2005;81(1):41-6. 